\title{
STUDIES OF THE CONNEXIONS OF THE FORNIX SYSTEM
}

\author{
BY \\ H. M. DAITZ and T. P. S. POWELL \\ From the Department of Human Anatomy, University of Oxford
}

During recent years, particularly since anatomical and physiological experimental methods have failed to provide evidence that the hippocampus has a direct relationship to olfaction (Rose and Woolsey, 1943 ; Fox, McKinley, and Magoun, 1944 ; Le Gros Clark and Meyer, 1947 ; Meyer and Allison, 1949), increasing attention has been focused on this cortical structure. Its functional significance remains obscure, but reports (Klüver and Bucy, 1938 ; Bard and Mountcastle, 1948) that its destruction may lead to profound disturbances of behaviour in experimental animals suggests that it serves an important function in relation to cortical activities as a whole. These observations also to some extent support the hypothesis, originally put forward by Papez (1937), that the hippocampus and its connexions are essential components of a neural mechanism underlying emotional reactions. In the attempt to elucidate the functional significance of the hippocampus it is a matter of some importance that its connexions should be established with reasonable certainty, and this can only be done by the use of experimental methods. Most of the statements on the hippocampus which are to be found in standard textbooks of neuro-anatomy are based on the study of normal material, but the difficulties of interpreting fibre connexions in such material are so great that conclusive results can but rarely be obtained. It has been reasonably well established that the efferent fibres of the hippocampus are collected together in the fimbria and fornix and are for the most part distributed to the septum and mammillary nuclei, and possibly, by way of the ventral psalterium, to the hippocampus of the opposite side. The fimbria thus appears to be a complex bundle containing projection and commissural fibres. The fibres are commonly assumed to be mainly the axons of the pyramidal cells of the hippocampus, but there is no certainty from which pyramidal cells or from which fields of the hippocampus these different components of the fornix system take their origin. Recent physiological studies by Stoll, Ajmone-Marsan, and Jasper (1951) have indicated the presence in the fornix system of fibres passing backwards from the septum and this is also suggested by the Marchi experiments of Morin (1950). If this is so, it becomes evident that the fornix system is even more complex in its fibre components than has generally been supposed.

Several experimental methods are available for studying the connexions of the fornix system. The Marchi technique has serious limitations because a high proportion of the fornix fibres are very fine. Silver methods demonstrating terminal degeneration have been used with some success by Sprague and Meyer (1950) and by Simpson (1952). The method of retrograde cell degeneration may be expected to give positive results if the degeneration is sufficiently well marked to be readily detected in Nissl preparations, and consistently present with the same type of lesion. So far as the hippocampus and fornix system are concerned the only previous study with this method is that of Allen (1944) who, in his brief report, described chromatolysis of the pyramidal cells of the hippocampus and of the polymorphic cells of the dentate gyrus in dogs after fornix section. In the present communication the method of retrograde degeneration has been applied to the study of the fornix system following a number of experimental lesions, and it has led to certain well defined results. The fibre degeneration which has resulted from these lesions will be described in detail subsequently.

\section{Material and Methods}

The animals used in these experiments were 20 albino rats, two rabbits, and one macaque monkey. The rats varied in age from four days to fully grown adults. For the fimbrial lesions a small trephine hole was made on one side of the skull just behind the midpoint between the anterior and posterior poles of the cerebral hemisphere. An ophthalmic knife was inserted to a depth estimated from preliminary studies to be sufficient to sever the fimbria. In one rat the fimbria was divided on both sides. The animals in most cases made a good recovery, and were allowed to survive for periods ranging from one week to 10 months. In one 
adult rat an attempt was made to destroy the amygdaloid nuclei. In order to do this the antero-lateral part of the skull was opened through the orbital wall and the cortex and underlying tissue in the region of the amygdala removed by suction. This animal was allowed to survive for five weeks. The rats were killed under ether anaesthesia and the vascular system perfused with saline followed by either $70 \%$ alcohol or a mixture of alcohol, formalin, and acetic acid (Bodian, 1937).

The experimental procedure was similar in the rabbits. The midparietal region of one cerebral hemisphere was exposed and an ophthalmic knife was inserted downwards and medially. After one month the animal was killed and the brain removed and fixed in $70 \%$ alcohol.

In the monkey the brain was exposed by raising an osteoplastic flap. After retracting the left cerebral hemisphere the corpus callosum was brought to view and incised sagittally to open the third ventricle. A rightangled hook was inserted through this incision and rotated to lie in the coronal plane. The hook was then withdrawn and in doing so the left fornix was completely divided. The animal made a good recovery ; after seven weeks it was killed and the brain was removed and fixed in $70 \%$ alcohol and $2 \%$ acetic acid.

From all the brains blocks containing the septum, thalamus, hypothalamus, amygdala, and hippocampus of both sides were embedded in paraffin wax. Sections were cut at $15 \mu$ or $20 \mu$ and mounted serially. They were stained either with Borrel's methylene blue or with protargol. Series of normal brains stained with methylene blue, protargol, and by the Golgi-Cox technique were used for comparison.

\section{Experimental Results}

The regions of the brain considered relevant for the study of retrograde degeneration following the fornix lesions included the hippocampus, septum, amygdala, thalamus, and hypothalamus. These were examined in methylene blue preparations for cell loss or degenerative change and in all cases of unilateral lesions strictly comparable levels on the two sides were always used for comparison.

Hippocampus. - Irrespective of the species, age of the animal, or the survival time, in all experiments there was a consistent absence of any detectable change in the cells of the hippocampus (except in the immediate proximity of the lesion). The most careful comparison of the cells in all fields of the hippocampus on each side showed no significant difference, either in their number, size, or staining properties ; nor was there any gliosis evident in the pyramidal cell layer on the operated side (Figs. 1-4). In order to confirm the impression that there was no cell loss on the operated side sample counts of the cells in the different fields of the hippocampus of both sides were made in one experiment. These were done with the use of a graticule inserted into a $\times 6$ eyepiece and a 0.65 N.A. objective. All the nerve cells in six corresponding fields, selected to sample the whole cross-sectional area, were counted on each side. This was repeated at five evenly spaced levels through the antero-posterior extent of the hippocampus. These counts showed no significant difference between the two sides. It is particularly to be noted that even in rats a few days old, and even after a survival time of 10 months after complete section of the fornix, no changes in the appearance or number of the cells of the hippocampus could be detected. Further, neither section of the dorsal fornix as well as the fimbria in one rat (H 57), nor bilateral division of the fimbria (D 225), caused any appreciable change in the hippocampal cells.

Although no cellular degeneration could be found in the hippocampus in this series of experiments, the alveus on the affected side in the rats was considerably reduced in thickness and showed a severe gliosis. Following division of the fimbria alone this degeneration involved the whole alveus over the ventral aspect and extended medially along the alveus on the dorsal aspect of the hippocampus from the fimbria to approximately the junction of the middle and medial thirds, that is to say, to a level between Lorente de Nó's fields $\mathbf{C A}_{1}$ and $\mathbf{C A}_{2}$. Sections stained with protargol demonstrated a severe loss of fibres in the lateral two-thirds of the alveus but there was no change in the medial third. On the other hand, following division of both the fimbria and dorsal fornix the alveus shrank and fibres were lost along its whole medio-lateral extent. It is of interest to note that examination of the normal side in protargol-stained sections shows that the fibres in the lateral two-thirds of the alveus on the dorsal surface of the hippocampus (and in the entire alveus over the ventral segment) are directed laterally to the fimbria whereas those in the medial third extend into the dorsal fornix. This divergence of the axons at the junction of fields $\mathrm{CA}_{1}$ and $\mathrm{CA}_{2}$ is also shown distinctly in sections of normal rat brains treated by the Golgi-Cox technique. No cell or fibre degeneration could be seen in the stratum oriens of the hippocampus in any of the rat brains following fornix lesions.

In the rabbits the alveus was not appreciably shrunken and there was no gliosis in methylene blue preparations. As the fornix was not completely divided (approximately a third being intact), and no protargol-stained sections were available, this finding is inconclusive. In the monkey, however, the alveus showed no detectable change either in methylene blue or protargol-stained sections though the fornix had been completely severed. 


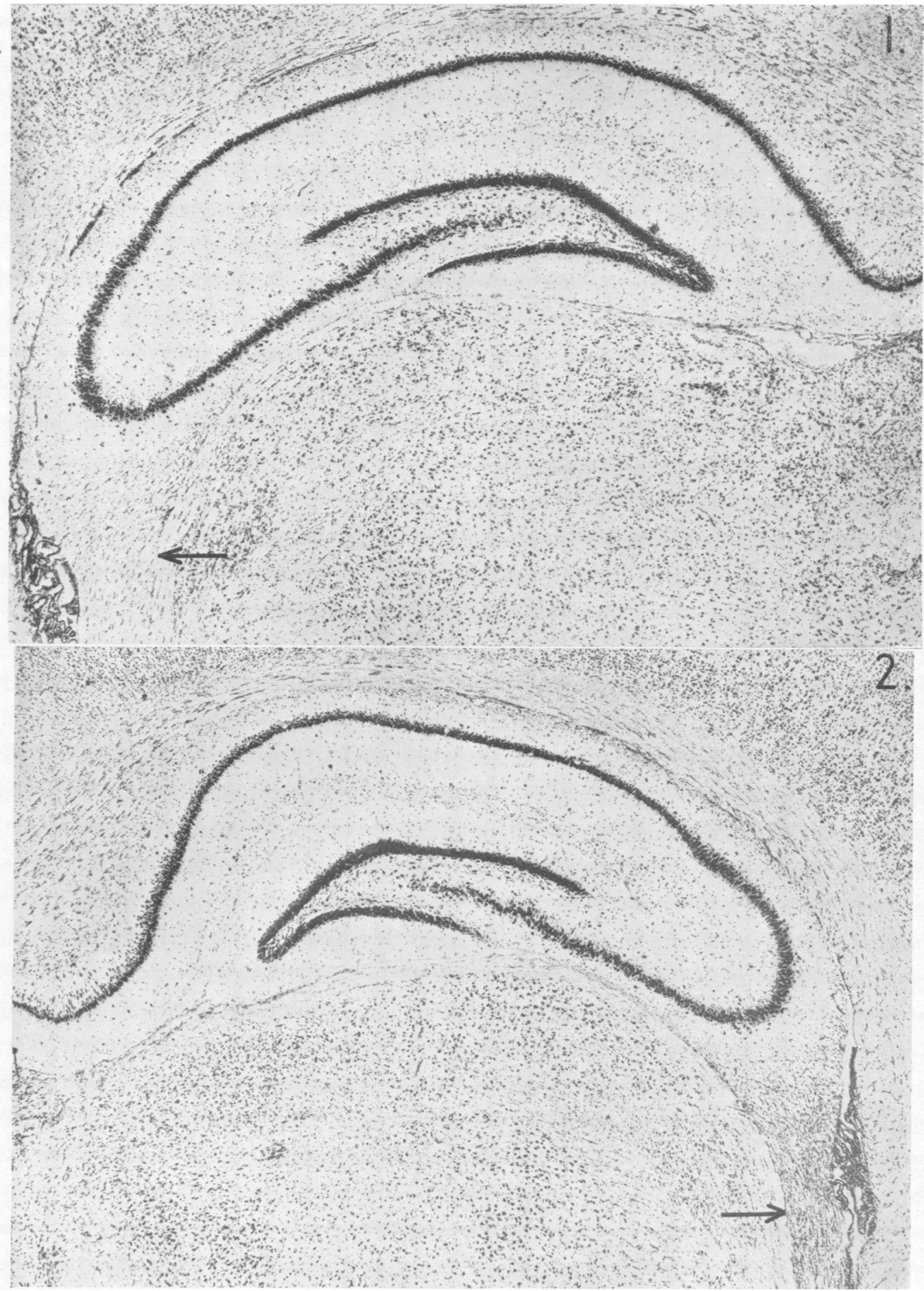

Fig. 1.- Coronal section showing the hippocampal formation on the unoperated side in rat $\mathbf{H} 50$. The normal fimbria is indicated by an arrow.

FIG. 2.-The hippocampal formation of the operated side in rat $\mathbf{H} 50$ three months after complete division of the fimbria. The latter (indicated by an arrow) is shown caudal to the site of the lesion; it is grossly degenerated and infiltrated with neuroglial cells. $\times 34$ 
The Septal Region : Medial Septal Nucleus.This nucleus consists of two elements, a small-celled anterior part composed of stellate cells with very little Nissl substance and a large-celled posterior part composed of two cell types, large multipolar cells rich in Nissl material and small ovoid cells. Following section of the fimbria the parvicellular element remains unchanged. The changes in the magnocellular part vary with the length of survival after the experimental lesion. After seven days the majority of the cells are shrunken and stain less intensely ; these changes are sharply limited by the midline and thus clearly demarcate the nuclei of the two sides. After two weeks some degree of cell-loss has occurred. One month after operation the magnocellular element of the medial septal nucleus on the operated side appears under low power to have disappeared completely and the nucleus of the normal side stands out in sharp contrast (Fig. 5). Examination under higher magnification shows that though many cells have disappeared a number do persist. Most of these are small, lightly staining cells, but there are also fusiform, pyknotic cells which have a shrunken appearance ; an occasional normal large cell is also present. After the longer survival periods of three and 10 months the shrinkage of the remaining large cells is even more marked and they practically all appear pyknotic and spindle-shaped. It is now exceptional to find a normal large cell, but the smaller, lightly staining cells are present. Only a slight degree of gliosis is seen even after 10 months. Essentially the same changes were found in the rabbit and monkey experiments.

In one experiment (D 208) with a survival of seven days, the stria terminalis was divided without involvement of the fimbria. No appreciable degeneration was found in the medial septal nucleus following this lesion.

Lateral Septal Nucleus.-This nucleus lies between the medial septal nucleus and the lateral ventricle. The peripheral part of the nucleus is composed of medium-sized ovoid and stellate cells which stain rather lightly ; in the medial part of the nucleus the cells are less closely packed and are smaller and stain more deeply. Following division of the fimbria there is severe neuroglial infiltration of the lateral septal nucleus and, in the medial sparsely populated area of the rat and the rabbit, the cells, while showing no cytological evidence of degeneration, are somewhat larger than normal and have a more rounded appearance ; they also stain less deeply than those on the normal side. These cells are now identical in appearance with those of the lateral part of the nucleus, both on the normal and the operated sides. This change first appears after two weeks, and the same appearance persists even after 10 months. Protargol-stained sections show that practically all the fibres that normally traverse the medial part of the lateral septal nucleus have disappeared. The changes in the cells of this area thus probably result from the fact that they are released from the mechanical compression normally exerted by the dense bundles of fibres which traverse the nucleus. It is certain that the difference between the cells of the two sides is not due to shrinkage of the cells on the unoperated side, for a comparison with series of normal brains shows that the cells on this side retain their normal appearance. Following bilateral division of the fimbria in the rat the cells in this part of the lateral septal nucleus become slightly enlarged on both sides.

After section of the fimbria in the monkey's brain the lateral septal nucleus has become heavily infiltrated with glial cells and its total width is considerably diminished, the cells being more tightly packed together. In contrast to the rats and the rabbits, however, no alteration in the morphology of the individual cells has occurred.

The consistent retrograde degeneration of the cells $\stackrel{\mathbb{}}{\mathrm{Q}}$ of the medial septal nucleus following lesions of the fimbria makes it evident that the latter contains axons derived from this nucleus. In order to determine whether these axons extend as far as the amygdaloid region, an attempt was made to destroy the amygdaloid complex in a rat (D 151) and the animal was allowed to survive for two months. Serial sections of the brain showed that almost the entire entorhinal cortex and the anterior part of the amygdala had been destroyed, the lesion also extending medially to the lateral preoptic region. More posteriorly the cortical nucleus is undercut throughout most of its medio-lateral extent and its cells stain feebly. The caudal parts of the cortical and medial nuclei, and the ventral tip of the lateral nucleus, remain intact. An apparently undamaged stria terminalis can be seen leaving these nuclei. The hippocampus had not been damaged at all. The only change in the septal region is a lighter staining of the most peripheral cells of the lateral septal nucleus, but this can be attributed to the direct effect of the lesion. Both the medial and lateral septal nuclei of the operated side have their normal cell population and there is no neuroglial infiltration. The number and morphology of the cells in the nucleus of the diagonal band also remain unchanged, as well as those of the anterior hippocampal nucleus and the bed nuclei of the anterior and hippocampal commissures. 


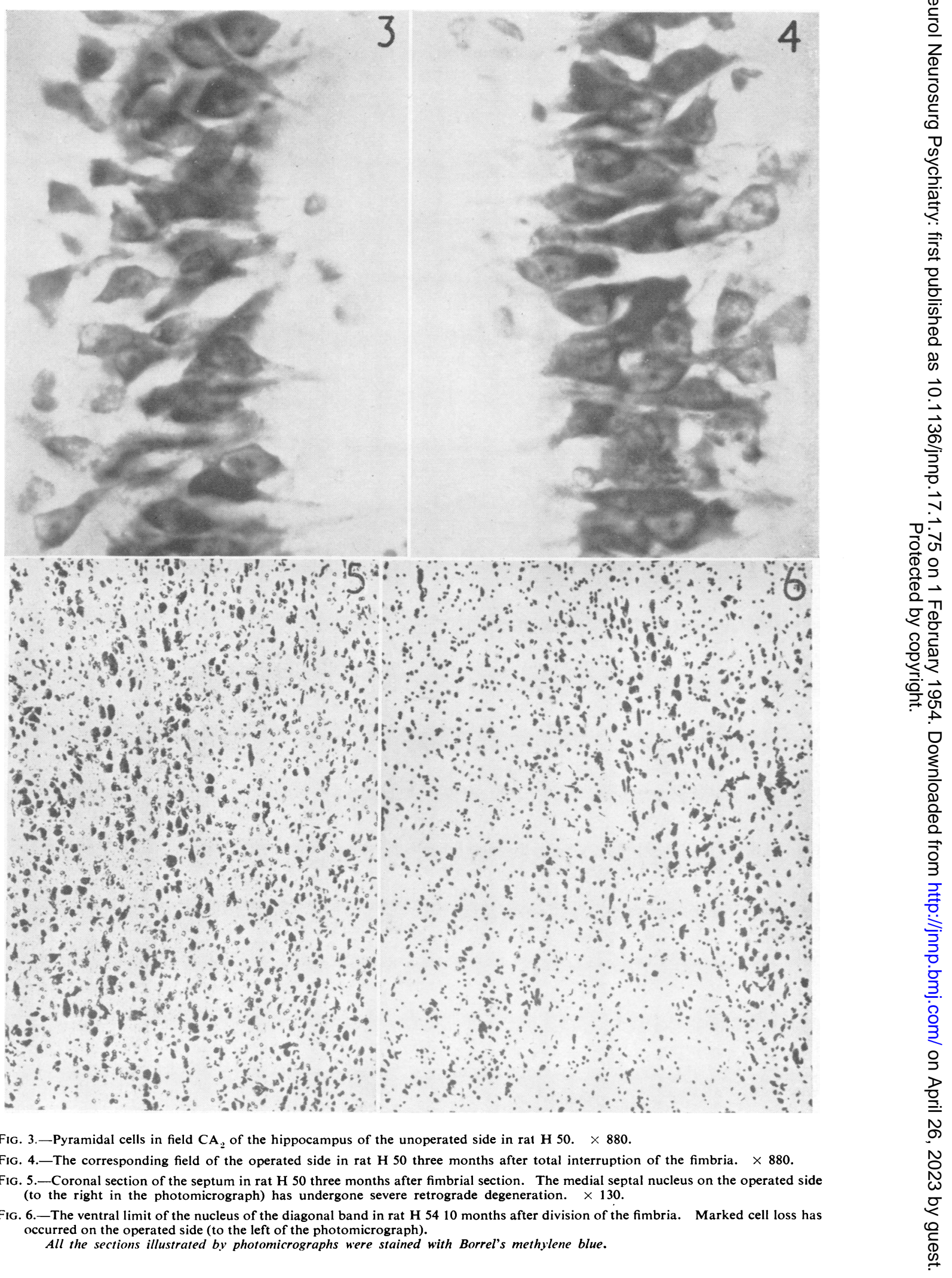


Triangular Nucleus of Cajal, Bed Nucleus of Stria Terminalis, Anterior Hippocampal Nucleus, Nucleus Accumbens.- None of these nuclei showed any significant change in any of the experimental animals.

Interstitial Nucleus of Ventral Psalterium.-The appearance of the cells of the interstitial nucleus in the rat and rabbit on the affected side of the commissure, and particularly in the degenerated dorsal fornix in $\mathrm{H} \mathrm{57}$, was noted both for the striking difference between the two sides, and for the enlargement of the cells which in their appearance come to resemble those of the lateral septal nucleus; as with the medial part of the lateral septal nucleus, this change is evidently a mechanical effect following loss of the fibres which normally penetrate among the cells. There is severe gliosis in the affected half of the commissure and protargol-stained sections show a marked loss of fibres. No change was found in the cells of the interstitial nucleus of the monkey ; this is related to the absence of any fibre degeneration in the ventral psalterium.

Nucleus of the Diagonal Band.-This is a prominent L-shaped bed nucleus in the telencephalon. Only the cells of the vertical limb of the nucleus on the operated side are affected by section of the fimbria. The reaction is very similar to that found in the medial septal nucleus, but it is not as complete or as uniform. After seven days a number of the cells have shrunk and stain less intensely. After longer survival periods many of the cells have completely disappeared but even after 10 months a proportion of normal cells persist (Fig. 6). It should be emphasized that division of the dorsal fornix in addition to the fimbria does not affect the amount of degeneration.

Bilateral section of the fimbria results in degeneration in the diagonal band nucleus on both sides. The severity and distribution of the degeneration in either nucleus is exactly the same as that after unilateral lesions. On the other hand, division of the stria terminalis alone, without involvement of the fimbria, does not lead to any degeneration (at least after seven days).

Pre-optic Region and Hypothalamus.-The different nuclei in these regions were examined carefully in all experiments, but, apart from that secondary to direct damage to the anterior thalamic nuclei, no degeneration was found.

Thalamus.--Inconstant changes have been found in some or all of the midline and intralaminar nuclei in many experiments. However, the appearance of the cells in any individual nucleus showed considerable variation in different animals ; sometimes they are swollen and stain less deeply, while in other experiments they are shrunken and pyknotic. There may or may not be accompanying cell loss. It has not been possible to correlate these variations either with the site and extent of the lesion in the fornix system or with the degree of thalamic involvement.

Epithalamus.-In one experiment, with a survival time of 15 weeks, in which the fimbria and stria terminalis had been divided (with involvement of the lateral thalamic nucleus) in a rat (H 50), the habenular nuclei of the operated side showed cytological changes. In the medial habenular nucleus all the cells had shrunk and stained more intensely, while those in the lateral habenular and parataenial nuclei were enlarged and stained more lightly as compared with the normal side. These changes were not accompanied by any cell loss or gliosis. Protargol preparations showed a distinct neuropil loss in these nuclei and the fibres of the stria medullaris were not so well impregnated as on the normal side. In other experiments with similar or more extensive lesions, and with the same or other survival times, no change was found in the habenular nuclei. The cell changes are thus not consistently related to the type of lesion and their significance in $\mathrm{H} 50$ remains undetermined.

The Amygdaloid Region.- - In those experiments in which the stria terminalis had been cut, each amygdaloid nucleus was examined in detail. Noô significant difference was detected either in the? number or morphology of the cells.

\section{Discussion}

Perhaps the most remarkable observation resulting from these experiments is the complete absence, following total interruption of the fornix system, of any demonstrable change in the cells of the hippocampus, either in the form of a cellular reaction in the short-term survivals or of cytological changes or cell loss in the long-term survivals. In view of the common assumption that the fornix fibres are derived mainly from the axons of the hippocampal pyramids and that they comprise the only efferent pathway of the hippocampus, this result was unexpected, the more so because of the statement by Allen (1944) that in the dog section of the fornix leads to chromatolysis of the hippocampal pyramids. Unfortunately, however, Allen did not support his statement by photomicrographs and it seems possible that he may have interpreted slight variations in the intensity of staining which are to be found in normal material as evidence of chromatolytic changes. 
Among the pyramidal cells, and in the stratum oriens and stratum radiatum of the hippocampus, and also in the polymorph layer of the dentate gyrus, we have found that occasional pale vacuolated cells are to be seen in normal brains.

The absence of cellular degeneration in the hippocampus in all our experiments is perhaps to be explained by the presence of a rich system of recurrent collaterals from the axons of the pyramidal cells. According to Lorente de Nó (1934) many of these axons are provided with conspicuous and branching collaterals of the Schaffer type which reach the stratum lacunosum, while others send off collaterals to form axial association pathways in the stratum radiatum. If Cajal is correct in his inference (1911) that the presence of such collaterals is responsible for the absence of retrograde degeneration of cells of the cerebral cortex following section of their main axons (the presence of these collaterals being assumed to be sufficient for maintaining an adequate physiological activity of the cells), it is possible that this is the explanation of the absence of any cytological change in the hippocampus following lesions of the fornix system. In Golgistained material axons are found to reach the alveus and it is certain that these fibres contribute to the fornix system, providing a projection pathway to the septal region and hypothalamus (cf. the experimental studies of Sprague and Meyer, 1950, and Simpson, 1952). Protargol-stained sections of our own experimental material show quite clearly that section of the fimbria in rats is followed by a conspicuous loss of fibres in the alveus, presumably due, at least in part, to the interruption of caudally directed fibres having their origin in the medial septal and diagonal band nuclei. When the lesion is confined to the fimbria the fibre loss does not involve the medial third of the dorsal part of the alveus ; this region is affected only if the dorsal fornix is interrupted. These observations provide clear evidence of differential connexions of different parts of the hippocampus with different components of the fornix system. An examination of Golgi-Cox and protargol sections of normal material also shows that the axons proceeding from the pyramidal layer of the hippocampus into the alveus diverge at the junction of the middle and medial thirds of its dorsal aspect, this divergence corresponding to the junction of fields $\mathrm{CA}_{1}$ and $\mathrm{CA}_{2}$. It is of some interest to note also that, according to Cajal (1911), Lorente de Nó (1934), and Adey and Meyer (1952), the afferent fibres to field $\mathrm{CA}_{1}$ are derived from the medial part of the entorhinal cortex by way of the temporo-alvear pathway, while those to the other fields of the hippocampus come from the lateral entorhinal cortex by way of the perforating tract of Cajal. It may be noted that in none of our experiments was any fibre loss detected in the other strata of the hippocampus. Further, no fibre degeneration was observed in the alveus of the monkey following complete division of the fornix ; however, this may be due to the fact that the lesion in this animal was anterior to the ventral psalterium, whereas in the rats the fimbria behind the psalterium was always involved.

In contrast to the total lack of cellular change in the hippocampus following fimbrial section is the severe, rapid, and consistent degeneration in the medial septal and diagonal band nuclei. There is no reason to doubt that this is a true retrograde degeneration, and it may be concluded therefore that a large proportion of the cells of these nuclei send their axons caudally into the fimbria. (It has already been noted that division of the stria terminalis by itself is not followed by any change in these nuclei.) The exact destination of these fibres remains uncertain, but the fact that the medial septal and diagonal band nuclei remain intact after extensive destruction of the entorhinal cortex and amygdaloid nuclei with preservation of the hippocampus strongly suggests that they terminate in the latter.

Previous authors have described a septo-ammonic tract on the basis of normal material (Crosby, 1917, in the alligator; Kappers, Huber, and Crosby, 1936, in mammals). Morin (1950) also traced Marchi degeneration into the fimbria following septal lesions in the guinea-pig. On the other hand, afferent fibres to the hippocampus from the septal areas by way of the fimbria have been denied by Cajal $(1901,1911)$ and Lorente de Nó (1934), and this opinion appears to be generally accepted.

Rose and Woolsey (1943) reported complete degeneration of the medial septal nucleus and degenerative changes in the "diagonal area" in rabbits after ablation of the greater part of the telencephalon, including the hippocampus; it is interesting to note, however, that in two of their experiments in which the hippocampus and fimbria had been spared (together with " the olfactory structures in front of the septum ") these nuclei remained intact. More recently, Stoll and others (1951) have claimed on the basis of electro-physiological studies that in the cat a two-way path exists between the septum and the tip of the temporal lobe and that these connexions pass bilaterally in the fimbria. So far as the nucleus of the diagonal band is concerned, the experiments of Gerebtzoff (1939), supported by those of Sprague and Meyer (1950), have suggested that ascending fibres in the dorsal fornix originate in this nucleus. We have not been 
able to confirm this particular point, for our own experiments indicate that the extent of the retrograde degeneration in the nucleus of the diagonal band is not affected by the inclusion of the dorsal fornix in the operative lesion.

Since the stria terminalis lies in such close relationship to the fimbria it is almost impossible to cut one without damaging the other, and in most of our experiments both have been interrupted by the lesion. There is evidence that the stria terminalis originates in certain of the amygdaloid nuclei and we have therefore taken the opportunity of examining the latter in our material for retrograde degeneration in the cells. The results were uniformly negative ; they are thus in agreement with those of Fox (1943) who was unable to detect any change following either lesions of the stria terminalis or lesions placed on the anterior, posterior, medial, or lateral margins of the amygdaloid complex. It seems probable, therefore, that the efferent connexions of the amygdaloid complex are not amenable to study by the method of retrograde cell degeneration.

It has been mentioned that in many of the rat experiments inconsistent and variable cellular changes were noted in the midline and intralaminar nuclei of the thalamus. It is not possible to explain these findings with any certainty but it may be relevant to recall that Rose and Woolsey (1943) reported cellular degeneration in the midline and intralaminar nuclei following removal of the entire telencephalon. However, in two of their experiments (RW 6 and RW 8) in which the septum, hippocampus, and olfactory structures were preserved these nuclei showed little or no degeneration.

\section{Summary}

The connexions of the fornix system have been studied experimentally in the rat, rabbit, and monkey by the method of retrograde cell degeneration. No cellular degeneration was found in the cells of the hippocampus after complete division of the fimbria or fornix.

These observations were consistent in animals of all ages and after survival periods varying from seven days to 10 months.

It is suggested that the absence of retrograde degeneration in the cells may be due to the rich system of recurrent collaterals derived from the proximal ends of the axons of the hippocampal pyramids.

Extensive fibre degeneration of the alveus occurs in the rat following lesions of the fornix system. From the distribution of this degeneration it appears that field $\mathrm{CA}_{1}$ of the hippocampus is related to the dorsal fornix and the other fields to the fimbria.

Division of the fimbria results in complete atrophy or shrinkage of all the cells of the ipsilateral medial septal nucleus and partial degeneration of the nucleus of the diagonal band. Additional involvement of the dorsal fornix or stria terminalis in the rat did not affect the degree or distribution of this degeneration.

No retrograde degeneration was found in the nuclei of the pre-optic and hypothalamic areas.

Extensive destruction of the entorhinal cortex and amygdaloid nuclei was not accompanied by any degeneration in the septal nuclei.

Division of the stria terminalis did not lead to any cellular change in the amygdaloid nuclei.

We wish to acknowledge our thanks to Professor W. E. Le Gros Clark for his constant advice and criticism. We would also thank Dr. D. A. Simpson for performing the operation on the monkey.

This work was done while one of us (T. P. S. P.) held a Medical Research Council Fellowship in Clinical Research.

\section{REFERENCES}

Allen, W. F. (1944). J. comp. Neurol., 80, 283.

Adey, W. R., and Meyer, M. (1952). Brain, 75, 358.

Bard, P.. and Mountcastle, V. B. (1948). Res. Publ. Ass. nerv. ment. Dis., 27, 362.

Bodian, D. (1937). Anat. Rec., 69, 153.

Cajal, S. Ramon y, (1901). Trab. Lab. Invest. biol. Univ. Madr., 1, 1-227. 1911). Histologie du système nerveux de l'homme et des vertébrés, vol. 2. Maloine, Paris.

Crosby, E. C. (1917). J. comp. Neurol., 27, 325.

Fox, C. A. (1943). Ibid., 79, 277.

Fox, McKinley, W. A., and Magoun, H. W. (1944). J. Neurophysiol., 7, 1.

Gerebtzoff, M. A. (1939). J. belg. Neurol. Psychiat., 39, 205.

Kappers, C. U. A., Huber, G. C., and Crosby, E. C. (1936). The Comparative Anatomy of the Nervous System of Vertebrates, including Man, vol. 2. Macmillan, New York.

Klüver, H., and Bucy, P. C. (1938). J. Psychol., 5, 33

Le Gros Clark, W. E., and Meyer, M. (1947). Brain, 70, 304.

Lorente de Nó, R. (1934). J. Psychol. Neurol., Lpz., 46, 113.

Meyer, M., and Allison, A.C. (1949). Journal of Neurolog.v, Neurosurgery and Psychiatry, 12, 274.

Morin, F. (1950). J. comp. Neurol., 92, 193.

Papez, J. W. (1937). Arch. Neurol. Psychiat., Chicago, 38, 725. Rose, J. E., and Woolsey, C. N. (1943). Bull. Johns Hopk. Hosp., 73, 65.

Simpson, D. A. (1952). Journal of Neurology, Neurosurgery and Psychiatry, 15, 79 .

Sprague, J. M., and Meyer, M. (1950). J. Anat., Lond., 84. 354. Stoll, J., Ajmone-Marsan, C., and Jasper, H. H. (1951). J. Neuro-
physiol., 14, 305 . 